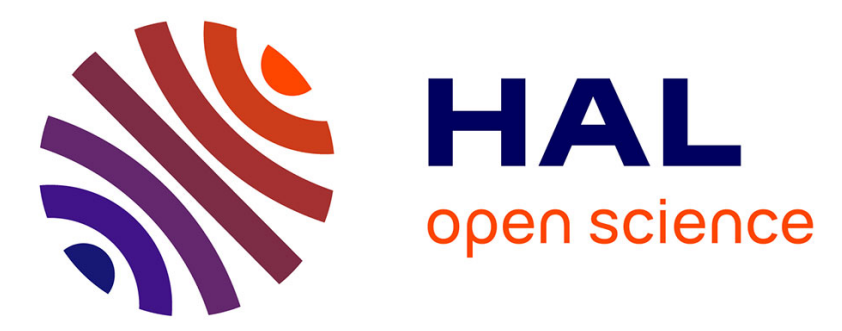

\title{
Louise Weiss et quelques autres : candidates et élues gaullistes au Parlement européen (1979-1989)
}

\author{
Yves Denéchère
}

\section{To cite this version:}

Yves Denéchère. Louise Weiss et quelques autres : candidates et élues gaullistes au Parlement européen (1979-1989). Histoire@Politique: revue du Centre d'histoire de Sciences Po, 2012, 17, pp.17-2012. halshs-01091653

\section{HAL Id: halshs-01091653 \\ https://shs.hal.science/halshs-01091653}

Submitted on 5 Dec 2014

HAL is a multi-disciplinary open access archive for the deposit and dissemination of scientific research documents, whether they are published or not. The documents may come from teaching and research institutions in France or abroad, or from public or private research centers.
L'archive ouverte pluridisciplinaire HAL, est destinée au dépôt et à la diffusion de documents scientifiques de niveau recherche, publiés ou non, émanant des établissements d'enseignement et de recherche français ou étrangers, des laboratoires publics ou privés. 


\section{Louise Weiss et quelques autres :}

\section{candidates et élues gaullistes au Parlement européen (1979-1989)}

\section{Yves Denéchère}

Dès les années du RPF et les premiers pas de la construction communautaire des femmes gaullistes soutiennent ce processus, contre l'avis du Général de Gaulle, qui les laisse néanmoins faire ce choix. C'est du moins ce que raconte Irène de Lipkovski (1898-1995), députée gaulliste, qui soutient la CECA contre l'avis du général de Gaulle : «Un jour il m'interrogea sur mes raisons de vouloir voter pour la Communauté charbon-acier à l'Assemblée ; lui, voulait que nous la repoussions. Moi, je pensais que nous ne devions pas avoir l'air d'être contre l'organisation de l'Europe. Il me laissa libre de voter suivant ma conscience. J'ai donc voté pour la CECA et je m'en suis réjouie plus $\operatorname{tard}^{1}$. »

La première élection au suffrage universel des députés européens en 1979 résulte d'un engagement du président Giscard d'Estaing. De Gaulle et Pompidou étaient très opposés à cette élection dans laquelle ils voyaient le début d'un abandon de souveraineté nationale. Sur l'Europe, l'opposition entre gaullistes et UDF est grande, voire grandissante. Le 6 décembre 1978, le célèbre « appel de Cochin », du nom de l'hôpital où Jacques Chirac est alors alité à la suite d'un accident de voiture, marque un point de non-retour. Le président fondateur du RPR y stigmatise le «parti de l'étranger », précisant quelques jours plus tard que l'on trouve des adeptes de celui-ci aussi bien dans la majorité que dans l'opposition. Ces déclarations suscitent des remous jusque dans les rangs du RPR. Alain Peyrefitte, ministre de la Justice du gouvernement Barre, pointe du doigt les «personnages occultes qui semblent s'être emparés » de l'appareil du RPR. Tous les observateurs identifient Charles Pasqua et les conseillers de Jacques Chirac: Pierre Juillet et Marie-France Garaud auxquels on prête la paternité de l'«appel aux Français ». La principale intéressée a souvent indiqué que ce texte était le fruit d'un travail collectif; que le terme «parti de l'étranger » était de Pierre Juillet, s'inspirant largement des Mémoires du général de Gaulle. Il visait tous les gens qui croyaient la France incapable de ne rien faire toute seule ${ }^{2}$. En 1979, le RPR se retrouve donc un peu dans la même situation que le PCF : devoir faire une campagne pour un scrutin européen qu'il n'a pas souhaité. La thématique européenne n'est pas non plus celle sur laquelle le parti est le plus à l'aise.

Dès ses premières années, le RPR n'est pas un parti où les femmes participent aisément à la vie politique. Pourtant, parmi les 81 noms de la liste proposée aux suffrages des

\footnotetext{
${ }^{1}$ Andrée Dore-Audibert et Annie Morzelle, Irène de Lipkowski, le combat humaniste d'une Française du XX siècle, Laval, Siloë, 1998, p. 163-164.

${ }^{2}$ Marie-France Garaud, La fête des fous. Qui a tué la Ve République ?, Paris, Plon, 2006.
} 
Français pour les élections européennes de 1979, il faut bien en faire figurer un certain nombre... Les deux scrutins européens suivants (1984 et 1989) sont très difficiles pour les femmes, en particulier pour les gaullistes qui sont bien peu nombreuses à être candidates et élues sur des listes qui regroupent plusieurs partis de l'opposition.

Grâce aux archives du Centre Féminin d'Etudes et d'Information (CFEI), créé en 1965 par le général de Gaulle, et d'autres fonds conservés au Centre des Archives du Féminisme (CAF) d'Angers, il est possible de mieux comprendre comment les femmes gaullistes ont cherché en vain à s'imposer comme candidates lors des trois premières élections européennes. La presse, la documentation du Bureau d'information à Paris du Parlement européen et des entretiens individuels réalisés au milieu des années 2000 dans le cadre d'une recherche inédite constituent les autres sources principales de cette étude ${ }^{3}$.

\section{I - 1979 : l'alibi Louise Weiss}

La liste du RPR intitulée Défense des Intérêts de la France en Europe (DIFE) est de loin la moins féminisée des quatre "grandes » listes puisqu'elle ne compte que 12 femmes sur 81 candidats. Les femmes socialistes ont elles obtenu qu'un tiers des candidats soient des candidates et ce par groupe de trois noms, c'est-à-dire qu'environ un tiers des élus socialistes devraient être des femmes. Les quatre premières places sont occupées par Jacques Chirac, Michel Debré, Pierre Messmer et Claude Labbé. Les femmes gaullistes du CFEI qui en 1974 prend le nom de CFEI - Femme Avenir ${ }^{4}$, sont présentes... mais loin derrière. Ainsi, en $44^{\mathrm{e}}$ position figure Christiane Papon-Eraud (née en 1927), présidente de l'association depuis 1975 et membre du comité central du RPR. Noëlle Dewavrin-Danes (1933-2001), très active au sein de l'association et très intéressée par les questions européennes, est elle à la $59^{\mathrm{e}}$ place. En avril 1979, elle était pourtant présentée par la presse comme «déjà prête » à siéger à Strasbourg ${ }^{5}$, sans doute a-t-elle été rétrogradée sur la liste - et de quelle manière ! - au dernier moment. En remontant vers le haut de la liste, on trouve en $33^{\mathrm{e}}$ position Magdeleine Anglade (1921-1998), membre du comité directeur du CNI (Centre National des Indépendants) et à la $22^{\mathrm{e}}$ place, Marie-Madeleine Fourcade (1909-1989), grande résistante, compagnon de la Libération.

Dans les places éligibles, en $10^{\mathrm{e}}$ position, Nicole Chouraqui-Dahan, plus jeune que les précédentes (1938-1987), incarne «une ascension politique éclair ${ }^{6} . »$ Amie de Françoise

\footnotetext{
${ }^{3}$ Yves Denéchère, Ces Françaises qui ont fait l'Europe, Paris, Audibert, 2007.

${ }^{4}$ Centre des Archives du Féminisme, Angers, fonds 9 AF Femme Avenir, notamment 9 AF 8 sur Christiane Papon et 9 AF 37 : Caroline Corren, CFEI Femme Avenir: un progrès vers une meilleure représentation politique féminine?, IEP Grenoble, direction de Maurice Croisat et Dominique Labbé, 1991, 196 p.

${ }^{5}$ Le Figaro Magazine, 13 avril 1979, « Neuf Françaises pour l'Europe des Neuf », portrait de Noëlle Dewavrin.

${ }^{6}$ Le Figaro Magazine, 13 avril 1979, « Neuf Françaises pour l'Europe des Neuf », portrait de Nicole Chouraqui.
} 
Giroud, déçue du radicalisme et de Jean-Jacques Servan-Schreiber à l'occasion des élections municipales de Paris en 1977, elle a été attirée au RPR par Marie-France Garaud. Lors de la campagne des élections législatives de 1978, elle fait une tournée dans 27 départements pour soutenir les candidats gaullistes et adhère ensuite au RPR où elle devient secrétaire générale adjointe. Jacques Chirac l'impose en bonne place sur la liste des européennes de 1979, «pour me renvoyer l'ascenseur », dit-elle simplement ${ }^{7}$.

Marie-Madeleine Dienesch (1914-1998), qui pointe au $7^{\mathrm{e}}$ rang de la liste, a au contraire sa carrière politique derrière elle. Après avoir été résistante au sein du réseau Libération Nord, elle a adhéré au MRP en 1945. C'est «l'une des rares femmes politiques à s'imposer durablement dans la vie politique française sous la $\mathrm{IV}^{\mathrm{e}}$ puis sous la $\mathrm{V}^{\mathrm{e}}$ République $^{8}$. » Elle est élue 11 fois consécutives à l'Assemblée nationale de 1946 à 1978 et est la première femme à présider une commission parlementaire en 1967-1968. Elle entame alors une carrière ministérielle comme secrétaire d'État chargée de différents portefeuilles dans quatre gouvernements (1968-1974). En 1975, elle est nommée ambassadrice au Luxembourg où elle reste trois ans ${ }^{9}$. En tant que vice-présidente internationale de l'Union Européenne Féminine, elle a particulièrement le profil pour figurer sur la liste des européennes.

Quant à la première femme sur la liste, il ne s'agit rien moins que de Louise Weiss (1893-1983) à qui Jacques Chirac a promis : «vous serez notre First Lady ! », avec la garantie de figurer dans les dix première places. Finalement, elle sera $5^{\mathrm{e}}$ et donc assurée de siéger au Parlement. Compte tenu de son âge, 86 ans, il apparaît très vite qu'elle en sera la doyenne et devra prononcer la première allocution. Dès avril 1979, elle se réjouit à l'avance de ce discours : « une volupté qu'elle ne laisserait pour rien au monde ${ }^{10} \ldots$ » Louise Weiss est dans la derrière partie de sa vie et reçoit récompenses, hommages, décorations et titres honorifiques. Rien que pour 1978, elle reçoit le prix Robert Schuman pour ses Mémoires d'une Européenne, et le prix de l'Europe décerné par le syndicat des journalistes et écrivains. Tous se souviennent qu'elle a dirigé la revue L'Europe Nouvelle au lendemain de la Grande Guerre et qu'elle a été une européiste convaincue, croyant en la réconciliation entre la France et l'Allemagne.

Fig. 1 Bulletin publicitaire et d'abonnement de L'Europe nouvelle,

\footnotetext{
${ }^{7}$ Thierry Desjardins, Les Chiraquiens, 1986, pp.261-272. Nicole Chouraqui est la seule femme parmi les seize chiraquiens présentés.

${ }^{8}$ Christian Bougeard, «Marie-Madeleine Dienesch : une carrière politique féminine méconnue », Clio, 1998, $\mathrm{n}^{\circ} 8, \mathrm{pp} .235-248$.

${ }^{9}$ Elle est la deuxième femme nommée ambassadeur après Marcelle Campana en 1972, voir Yves Denéchère, «Les ambassadrices: des femmes dans un territoire d'hommes », in Christine Bard (dir.), Le genre des territoires, 2004, pp.111-120.

${ }^{10}$ L'Aurore, 12 avril 1979, « la "First Lady" de Chirac ».
} 
papier imprimé, collection particulière, (C) DR.

Elle-même a créé en 1971 une fondation qui porte son nom et qui décerne un prix annuel pour prolonger son action en faveur de l'unité européenne et de l'avancement des sciences de la paix ; en 1978, elle le remet personnellement au chancelier Helmut Schmidt et en 1980 au Président Anouar El Sadate, au Caire ${ }^{11}$. Louise Weiss apparaît en quelque sorte comme la caution européenne de Jacques Chirac, sa présence sur la liste prouvant que l'on peut être européen-ne et opposé-e aux orientations choisies pour la construction européenne. Pourtant et justement, son engagement européen, mais aussi ses combats féministes ne cadrent pas vraiment avec les options politiques et les pratiques internes du RPR. Aussi, cet atout dans le jeu de Jacques Chirac se révèle être parfois une carte encombrante car les propos de Louise Weiss peuvent brouiller le message très anti-européen de Jacques Chirac et Michel Debré. Ne proclame-t-elle pas que «les différences entre les visions européennes du Président de la République et de Monsieur Chirac ne sont pas aussi importantes qu'on le dit $^{12}$. » C'est évidemment dommageable lorsque le RPR fonde toute sa campagne sur l'opposition à Valéry Giscard d'Estaing. D'un autre côté, il ne faut sans doute pas exagérer l'impact des déclarations - peu nombreuses - de Louise Weiss au cours de la campagne électorale.

La femme la plus importante de la campagne du RPR est en fait... Simone Veil, tête de liste UDF. Avant même le début de la campagne, Pierre Charpy dans La Lettre de la Nation, organe du RPR, fait preuve du machisme le plus grossier à l'égard de la ministre de la Santé : «Simone Veil, tête de liste pour l'élection européenne ? Comment pouvez-vous imaginer cela ? Pour qui l'a entendue au Club de la Presse d'Europe 1 dimanche soir, elle n'est qu'une aimable flâneuse dans ce jardin européen, qui joue de son ombrelle en espérant vaguement qu'il y aura quelques pommes à croquer pour Ève ${ }^{13}$. » C'est la reprise d'un « argument » vieux comme la volonté des hommes d'éloigner les femmes de la politique et du pouvoir : elles ne sont pas à leur place dans ce monde. Pis, elles ne peuvent apporter que le malheur aux hommes - politiques - à l'instar d'Ève déclenchant la colère divine. De son côté, Simone Veil avance que les positions des uns et des autres ne sont pas très éloignées : «quand je relis les déclarations du RPR en ce qui concerne l'Europe, je n'arrive pas à voir les divergences ${ }^{14}$. » Louise Weiss a déjà déclaré la même chose et refusé le combat : «WeissVeil, il y en a qui prennent des assonances pour des dissonances! J'ai trop de respect pour la

\footnotetext{
11 «La Fondation Louise Weiss et ses Prix » in Louise Weiss l'Européenne, Lausanne, Fondation Jean Monnet, Centre de recherche européennes, 1994, pp.441-453.

${ }^{12}$ Le Monde, 14 avril 1979.

${ }^{13}$ La Lettre de la Nation, 24 avril ; Le Monde, 24 avril 1979, « La Lettre de la Nation : Mme Veil, une aimable flâneuse ».

${ }_{14}$ «Club de la presse » d'Europe $n^{\circ} 1$, dimanche 22 avril 1979; Le Monde, 24 avril 1979, « Mme Veil souhaite que sa liste recueille $30 \%$ des suffrages ».
} 
personne et le patriotisme de Simone Veil pour accepter ce rôle d'adversaire ${ }^{15}$. » Mais pour Jacques Chirac: «sur l'Europe, ce qui nous sépare de l'UDF est incomparablement plus profond que ce qui nous unit ${ }^{16}$. »

\section{II - Un discours pour l'histoire, l'esprit partisan, et le « tourniquet »}

Avec trois femmes sur les 15 élus, le RPR a le même pourcentage d'élues que la liste menée par Simone Veil, mais nettement moins la liste des socialistes ${ }^{17}$. Louise Weiss, MarieMadeleine Dienesch - les deux députées françaises les plus âgées - et Nicole Chouraqui siègent au sein du groupe des Démocrates européens de progrès (DEP) qui rassemble les députés gaullistes et quelques Irlandais très hostiles à l'intégration européenne.

\section{Tableau 1 Les Françaises élues au Parlement européen en 1979} (par ordre de présentation sur les listes)

\begin{tabular}{|c|c|c|}
\hline Prénom Nom & $\begin{array}{c}\text { Date de } \\
\text { naissance }\end{array}$ & Profession et/ou responsabilité politique \\
\hline \multicolumn{3}{|l|}{ Liste Union de la France en Europe (UDF) } \\
\hline Simone Veil & 1927 & $\begin{array}{c}\text { Magistrate, } \\
\text { ministre de la Santé et de la Famille }\end{array}$ \\
\hline Christiane Scrivener & 1925 & $\begin{array}{c}\text { Chef d'entreprise, } \\
\text { ancienne secrétaire d'Etat à la Consommation }\end{array}$ \\
\hline Louise Moreau & 1921 & $\begin{array}{l}\text { Administratrice de sociétés, } \\
\text { député des Alpes-Maritimes }\end{array}$ \\
\hline Marie-Jane Pruvot & 1922 & Directrice d'école maternelle \\
\hline Simone Martin & 1943 & $\begin{array}{l}\text { Secrétaire de la Chambre d'agriculture } \\
\text { de Haute-Marne }\end{array}$ \\
\hline \multicolumn{3}{|l|}{ Liste PS } \\
\hline Edith Cresson & 1934 & Ingénieur en économie agricole \\
\hline Marie-Claude Vayssade & 1936 & Animatrice d'un centre de formation ouvrière \\
\hline Françoise Gaspard & 1945 & $\begin{array}{c}\text { Conseillère de tribunal administratif, } \\
\text { maire de Dreux }\end{array}$ \\
\hline Gisèle Charzat & 1941 & Enseignante \\
\hline Yvette Roudy & 1929 & Journaliste \\
\hline Yvette Fuillet & 1923 & Conseillère municipale de Marseille \\
\hline \multicolumn{3}{|l|}{$\begin{array}{c}\text { Liste Défense des Intérêts de la France en } \\
\text { Europe (RPR) }\end{array}$} \\
\hline Louise Weiss & 1893 & Ecrivaine, fondatrice de l'Ecole de la Paix \\
\hline Marie-Madeleine Dienesch & 1914 & $\begin{array}{c}\text { Député des Côtes-du-Nord, } \\
\text { Ancienne ministre } \\
\end{array}$ \\
\hline
\end{tabular}

\footnotetext{
${ }^{15}$ L'Aurore, 12 avril 1979, « la "First Lady" de Chirac ».

${ }^{16}$ Meeting du parc de Bagatelle, Paris, 2 juin 1979.

${ }^{17}$ La liste de Simone Veil est arrivée en tête avec 27, $6 \%$ des suffrages (25 élus), devant la liste socialiste (23,5\%, 22 élus) et la liste communiste (20,5\%, 19 élus). La liste du RPR obtient quant à elle 16,3\% des suffrages et 15 sièges.
} 


\begin{tabular}{|c|c|c|}
\hline Nicole Chouraqui & 1938 & Directrice d'un centre féminin de formation \\
\hline Liste PCF & 1943 & $\begin{array}{c}\text { Ouvrière soudeuse, } \\
\text { membre du Comité central }\end{array}$ \\
\hline Jacqueline Hoffmann & 1939 & $\begin{array}{c}\text { Conseillère générale du Var, } \\
\text { membre du Comité central }\end{array}$ \\
\hline Danielle De March & 1946 & $\begin{array}{c}\text { Adjointe au maire de Brest, } \\
\text { chercheuse scientifique }\end{array}$ \\
\hline Sylvie Le Roux & 1937 & Institutrice, membre du Comité central \\
\hline
\end{tabular}

Quelques jours avant la première réunion du Parlement européen nouvellement élu au suffrage universel à Strasbourg, Louise Weiss envoie à Valéry Giscard d'Estaing le texte du discours qu'elle entend prononcer ${ }^{18}$. Elle assure personnellement les traductions en anglais et en allemand ${ }^{19}$. Des proches, Andrée et Hubert Martin, témoignent : « elle y mit toute son âme, toute sa force. C'est près de nous qu'elle le prépara avec un dynamisme, une vitalité, une ardeur qui nous ahurissaient. C'était pour elle un couronnement ${ }^{20}$. » Le titre de son discours, «Un combat pour l'Europe » rappelle ceux qu'elle mena dans l'entre-deux-guerres et dont la relation est rééditée en $1979^{21}$.

Fig. 2 L'hémicycle européen « féminisé » (revue Euroforum), anonyme, septembre 1979,

(C) Centre des Archives du Féminisme, droits réservés.

Á 86 ans, Louise Weiss monte à la tribune «en amoureuse de l'Europe », «pour y vivre, présidente d'un jour, un honneur dont je n'aurais pas osé rêver, et une joie - la joie la plus forte que puisse éprouver une créature au soir de son existence -, la joie d'une vocation de jeunesse miraculeusement accomplie ». Dans son long discours, elle rappelle de nombreuses étapes de l'histoire de l'Europe et évoque des grandes figures, notamment les «trois grands Karl »: Charlemagne, Karl Marx et Charles de Gaulle ! Elle fait aussi entrer dans son Panthéon européen Jean Monnet et Robert Schuman, Winston Churchill et Konrad Adenauer. Evoquant l'élection du Parlement européen, Louise Weiss fait la leçon : «mes Européens chéris, avouez que vos campagnes électorales ont souvent paru plus lourdes d'arrières pensées partisanes que de préoccupations européennes ». Elle insiste sur le suffrage universel qui consacre la nouvelle assemblée : «je dis bien le suffrage universel, car les femmes y ont eu la part de plein droit qui leur revenait», et elle «salue chaleureusement celles qui se trouvent parmi nous, conscientes, sans esprit de ségrégation, de la tâche qui les

\footnotetext{
${ }^{18}$ Archives Nationales, Archives du président Giscard d'Estaing, 5 AG 3 / JR 8, élections européennes de 1979, correspondance, lettre de Louise Weiss à Valéry Giscard d'Estaing, 9 juillet 1979

${ }^{19}$ Le Figaro, 17 juillet 1979, « Lever de rideau à Strasbourg ».

${ }^{20}$ Andrée et Hubert Martin, «Louise Weiss toujours avec nous », in Louise Weiss l'Européenne, op. cit. ., p. 456.

${ }^{21}$ Louise Weiss, Mémoires d'une Européenne, tome II : Combats pour l'Europe 1919-1934, Albin Michel, 1979.
} 
attend ». Quant à l'avenir de l'Europe, pour Louise Weiss, il se résume à trois problèmes cruciaux : l'absence trop grande encore d'identité européenne, la dénatalité (« au train où vont les couples, il n'y aura plus d'Européens bientôt»), et les droits de l'homme. La doyenne termine «cette allocution si peu conforme aux usages », en mettant tout son espoir dans le Parlement européen ${ }^{22}$.

Globalement la presse française et européenne salue la performance de Louise Weiss. Des témoins se souviennent du discours de la doyenne des députés comme d'un moment fort de la séance inaugurale. Recevant le prix Louise Weiss 1988, Jacques Delors se souvient de la joie qui rayonnait sur son visage lorsqu'elle a appelé à la réinvention de l'Europe plurielle et solidaire et l'émergence d'une Europe des citoyens ${ }^{23}$. En revanche, Simone Veil n'évoque pas ce discours qui pourtant précède immédiatement son élection comme présidente du Parlement européen. Les relations entre les deux Françaises, aussi courtoises et respectueuses soientelles, sont assez tendues depuis le débat de la loi sur l'IVG. Le jour même de la séance inaugurale à Strasbourg, paraît une interview de Louise Weiss dans Le Matin. Elle répète qu'elle est opposée à l'avortement et n'est pas d'accord avec la loi Veil : «une loi de circonstance, un acte qui rend service aux femmes mais supprime la mauvaise conscience des hommes ». Au passage, elle se permet de qualifier Simone Veil de «jeunette ${ }^{24}$, formule peut-être inspirée par le désagrément d'avoir à partager les honneurs de la première séance du Parlement avec une autre femme, une autre Française.

Après le discours inaugural de Louise Weiss, dont la portée est essentiellement symbolique, sur le plan politique les choses sérieuses débutent avec l'élection à la présidence du Parlement. Elle se fait par vote secret, la majorité absolue des suffrages exprimés est nécessaire pour les trois premiers tours; au (dernier) tour suivant, la majorité simple est suffisante. Outre Simone Veil, se présentent le communiste Giorgio Amendola et le socialiste Mario Zagari. In extremis, deux autres candidats se présentent : un-e troisième Italien-ne : la radicale Emma Bonino et un deuxième Français : Christian de La Malène, chef du groupe DEP (RPR). Jacques Chirac ne cache alors pas que la candidature RPR - qu'il ne semblait pas envisager quelques jours plus tôt - va obliger Simone Veil à se rendre compte des déperditions de voix dans les groupes libéral, démocrate-chrétien et conservateur. Libération évoque la candidature La Malène comme «une peau de banane sous les pieds de la prétendante $^{25}$. $\gg$ Effectivement, elle empêche l'élection au premier tour de la candidate du parti libéral à laquelle il manque 8 voix. Les élus DEP/RPR ont réussi leur coup : ils

\footnotetext{
${ }^{22}$ Texte intégral du discours de Louise Weiss, dans Louise Weiss l'Européenne, op. cit., pp.481-502 ; Nombreux extraits dans Le Monde, 18 juillet 1979, «Mme Weiss appelle les parlementaires "à se saisir des problèmes cruciaux : identité européenne, natalité et droits de l'homme" ».

${ }^{23}$ Louise Weiss l'Européenne, op. cit. p.399 et entretien de l'auteur avec Jacques Delors le $1{ }^{\mathrm{er}}$ février 2005.

${ }^{24}$ Elle renouvelle ses critiques à l'égard de la loi Veil quelques mois plus tard dans Paris-Match, 9 novembre 1979, « Carte blanche à Louise Weiss ».

${ }^{25}$ Libération, 18 juillet 1979.
} 
démontrent ainsi que la coalition libérale, démocrate-chrétienne et conservatrice n'a pas la majorité. Cette manœuvre fragilise déjà Simone Veil : ce sont environ 25 voix de la coalition qui lui ont fait défaut. C'est le résultat de toutes les oppositions suscitées par la candidature Veil. La principale intéressée ne retient que l'absence de quelques voix en raison de 1'IVG ${ }^{26}$. Après le retrait de Christian de La Malène (et d'Emma Bonino), Simone Veil ne gagne que neuf voix au second tour et ne dépasse la majorité absolue que de trois voix. Nul doute que tous les suffrages obtenus par Christian de La Malène au premier tour ne se sont pas reportés sur Simone Veil. Ce qui n'empêche pas celui-ci de déclarer le lendemain : «C'est mon retrait au second tour qui a permis cette élection. [...] Il faut montrer que nos voix sont indispensables et que les gens qui prétendaient avoir la majorité dans cette Assemblée ne l'ont $\operatorname{pas}^{27}$.»

Fig. 3 Louise Weiss transmet les pouvoirs à Simone Veil au Parlement européen, 17 juillet 1979, anonyme, (C) Médiathèque de la Fondation Jean Monnet pour l'Europe, Lausanne.

Afin de montrer le peu de cas qu'il fait des mandats européens, Le RPR a institué le «tourniquet »: les élus ne siègeront que pendant une année et laisseront ensuite la place à leurs co-listiers situés derrière eux. Ainsi, Nicole Chouraqui et Marie-Madeleine Dienesch ne siègent que de juillet 1979 à octobre 1980. Le système a pour effet de multiplier les mandats d'un an et donc le nombre d'élu-e-s. Si les élues de juin 1979 font les frais du «tourniquet» d'autres femmes placées assez loin sur la liste accèdent au Parlement européen comme par exemple Magdeleine Anglade (33 ${ }^{\mathrm{e}}$ position) qui siège à Strasbourg en 1982-1983. Seule Louise Weiss, à qui le RPR ne peut pas imposer quoi que ce soit, refuse le «tourniquet » qui déconsidère l'élection au suffrage universel et empêche tout réel investissement de la part des élu-e-s. Elle demeure députée européenne jusqu'à sa mort en 1983.

\section{III - 1984 et 1989 : quelques égarées}

Les élections de 1984 et 1989 sont très difficiles pour les Françaises qui se sont beaucoup investi au Parlement européen. Les élues de droite doivent non seulement être capables de convaincre les appareils qu'elles ont le droit de retourner au parlement après y avoir bien travaillé - ce qui est également le cas pour les socialistes - mais elles doivent en plus faire face à une concurrence des hommes défaits lors des élections législatives de 1981 et 1988. Ce contexte explique que bien peu de femmes gaullistes participent à ces deux élections européennes.

\footnotetext{
${ }^{26}$ Entretien de l'auteur avec Simone Veil le 19 mars 2003.

${ }^{27}$ France Inter, 18 juillet 1979.
} 


\section{1- 1984 : deux élues RPR dans l'union de l'opposition}

Marie-France Garaud, qui a été candidate à l'élection présidentielle de 1981 et qui a fondé un Institut de Géopolitique en 1982, renonce à participer aux élections européennes de 1984 parce qu'elles «se présentent non comme un débat européen, sauf peut-être pour Simone Veil, mais comme un sondage grandeur nature sur le poids des partis politiques en France ». Au nom de la souveraineté de la France, Marie-France Garaud lutte contre toute idée d'intégration européenne et dénie au Parlement européen toute autorité : «si j'étais élue à l'Assemblée européenne, la première chose que je ferai, c'est de démissionner ${ }^{28}$. »

Comme l'indique Marie-France Garaud, Simone Veil entend mener une campagne véritablement européenne, mais l'union de l'opposition qui s'est imposée face à la majorité implique une forte dimension nationale. En février, elle affirme que la « liste unique permet bien davantage de faire passer le message européen », ce dont on peut évidemment douter après les positions des élus RPR au Parlement européen pendant la première législature ${ }^{29}$. Et effectivement, Simone Veil apparaît un peu comme l'otage politique du RPR dans cette campagne, tandis que le Front National la présente comme étant une femme de gauche...

En 1984, elle attaque le gouvernement de gauche, appelle à voter utile, stigmatise les petites listes et finalement parle peu d'Europe, en tout cas avec prudence. L'entrée de l'Espagne ? Elle serait «prématurée » car «admettre un pays dont on sait que la venue coûtera cher, c'est une imprudence »; autant dire qu'elle adopte la position du RPR de Jacques Chirac. Ce «mimétisme chiraquien » est dénoncé jusqu'à l'UDF, notamment par François Léotard, et apparaît à certains comme un chemin de Damas.

Les gaullistes ont choisi de donner un second mandat à Nicole Chouraqui, victime du «tourniquet» dès 1980. Á la $10^{\mathrm{e}}$ place, elle est la deuxième femme de la liste. Les autres gaullistes sont Magdeleine Anglade, qui contrairement à la précédente a bénéficié du «tourniquet » en 1982 , en $34^{\mathrm{e}}$ position, et, placée en $24^{\mathrm{e}}$ position, Anne-Marie Dupuy (19202008), qui s'est engagée dans les troupes d'Afrique du Nord pendant la Seconde Guerre mondiale et en est sortie avec le grade d'aspirant. Elle a lié son destin à celui de Georges Pompidou, en étant son chef de cabinet à Matignon puis son directeur de cabinet à l'Élysée. Elle vient d'être élue maire de Cannes en 1983. Dans ses mémoires, elle ne fait aucune allusion aux élections européennes de $1984^{30}$.

Au soir du 17 juin, 17 femmes sont élues députées européennes, une de moins qu'en 1979, mais les Française demeurent les plus nombreuses juste devant les Allemandes (16). La

\footnotetext{
${ }^{28}$ Libération, 8 décembre 1983 ; Le Matin, 9 décembre 1983.

${ }^{29}$ Le Quotidien de Paris, 9 février 1984, « Simone Veil, une campagne à la fois nationale et européenne ».

${ }^{30}$ Anne-Marie Dupuy, Le destin et la volonté. De Matignon à la mairie de Cannes, Paris, La Table ronde, 1996.
} 
détermination des femmes à figurer à des places éligibles sur les listes, notamment à gauche, a été payante.

Tableau 2 Les Françaises élues au Parlement européen en 1984 (par ordre de présentation sur les listes)

\begin{tabular}{|c|c|c|}
\hline Prénom Nom & $\begin{array}{c}\text { Date de } \\
\text { naissance }\end{array}$ & Antécédents européens \\
\hline \multicolumn{3}{|l|}{ Liste de l'Union de l'Opposition } \\
\hline Simone Veil & 1927 & $\begin{array}{l}\text { Elue en 1979, Présidente du Parlement 1979-1982 } \\
\text { Présidente de la commission juridique 1982-1984 }\end{array}$ \\
\hline Nicole Chouraqui (RPR) & 1938 & Elue en 1979, députée européenne 1979-1980 \\
\hline Nicole Fontaine & 1942 & \\
\hline Anne-Marie Dupuy (RPR) & 1920 & \\
\hline Christiane Scrivener & 1925 & $\begin{array}{l}\text { Elue en 1979, députée européenne 1979-1984; } \\
\text { rapporteur du budget pour } 1984 .\end{array}$ \\
\hline Magdeleine Anglade (CNI) & 1921 & Députée européenne 1982-1983 \\
\hline Jacqueline Thome-Patenôtre & 1906 & Membre de l'Assemblée 1958-1959 \\
\hline Simone Martin & 1943 & Elue en 1979, députée européenne 1979-1984 \\
\hline \multicolumn{3}{|l|}{ Liste PS } \\
\hline Nicole Péry & 1943 & Députée européenne 1981-1984 \\
\hline Gisèle Charzat & 1941 & Elue en 1979, députée européenne 1979-1984 \\
\hline Yvette Fuillet & 1923 & Elue en 1979, députée européenne 1979-1984 \\
\hline Marie-Claude Vayssade & 1936 & Elue en 1979, députée européenne 1979-1984 \\
\hline Colette Gadioux & $19--$ & \\
\hline Marie-Noëlle Lienemann & 1951 & \\
\hline \multicolumn{3}{|l|}{ Liste PCF } \\
\hline Danielle De March & 1939 & $\begin{array}{l}\text { Elue en 1979, députée européenne 1979-1984, } \\
\text { vice-présidente du Parlement }\end{array}$ \\
\hline Jacqueline Hoffmann & 1943 & Elue en 1979, députée européenne 1979-1984 \\
\hline \multicolumn{3}{|l|}{ Liste Front National } \\
\hline Martine Lehideux & 1933 & \\
\hline
\end{tabular}

Globalement, les femmes consolident leur place au Parlement européen : elles étaient 71 sur $434^{31}$ à la fin de la première législature, elles sont 75 au début de la deuxième. En France, avec plus de $43 \%$ des suffrages exprimés, la liste de Simone Veil remporte la majorité des sièges soit $41^{32}$. Le résultat est à peine à la hauteur des espérances de l'opposition qui avait envoyé en 1979 au Parlement européen 40 élus. Parmi les 41 élus, on compte huit femmes soit un taux de 19,5\% à peu près égal à celui des listes UFE et DIFE de 1979 (20 \%).

\footnotetext{
${ }^{31}$ Il faut ajouter aux 410 députés en 1979 les 24 représentants de la Grèce arrivés lors de l'entrée de leur pays dans la Communauté.

${ }^{32}$ La liste socialiste a recueilli $20,7 \%$ des voix (20 sièges), la liste communiste $11,2 \%$ (10 sièges) et la liste du Front National 10,95\% (10 sièges).
} 
Avec six femmes sur 20 députés, le PS augmente le taux de féminisation de sa délégation par rapport à 1979 (30\% contre 27,3\%). On compte seulement deux élues gaullistes. Au Parlement européen qu'elle retrouve - après quatre ans d'absence dus au «tourniquet »-, Nicole Chouraqui consacre son action aux problèmes de la famille et aux relations entre la C.E.E. et Israël. Anne-Marie Dupuy fait partie de la commission de l'environnement, de la santé publique et de la protection des consommateurs. Toutes les deux - ainsi que Magdeleine Anglade (CNI) réélue en 1984 - siègent au sein du groupe du Rassemblement des Démocrates Européens qui prend la suite du groupe DEP. Nicole Chouraqui en est l'une des viceprésidents. De janvier 1986 à octobre 1987, il se nomme Groupe des Rénovateurs et du Rassemblement des Démocrates Européens, avant de redevenir le Rassemblement des Démocrates Européens.

\section{2 - 1989 : des élues surtout rouges et vertes}

Les troisièmes élections européennes au suffrage universel de juin 1989 n'ont plus le caractère pionnier des deux premières éditions. Autre différence, situées un an après la présidentielle et les législatives de 1988 et juste dans la foulée des municipales de mars 1989, elles sont loin de toute échéance nationale importante. En 1989, ne croyant plus «à la dynamique de l'union » comme en 1984, Simone Veil souhaite une liste au centre, «la plus large possible » et ouverte aux rénovateurs de la droite ${ }^{33}$. Après bien des tractations et des difficultés autour de sa personne, Simone Veil prend la tête d'une liste $\operatorname{CDS}^{34}$, avec quelques rénovateurs, mais qui finalement se révèle assez étriquée face à une liste UDF-RPR conduite par Valéry Giscard d'Estaing et Alain Juppé dans laquelle il faut bien chercher les femmes en position éligible. Parmi les 16 femmes de la liste, seules Michèle Barzach et Michèle AlliotMarie en $4^{\mathrm{e}}$ et $6^{\mathrm{e}}$ places sont sûres d'être élues.

Seule femme ministre du gouvernement Chirac de cohabitation (1986-1988) avec le portefeuille de la Santé et de la Famille, Michèle Barzach (née 1943) est une figure féminine montante au sein du RPR. En 1988 elle a été élue députée de Paris et participe au courant des rénovateurs de l'opposition parlementaire. C'est d'ailleurs aussi pour cette raison qu'elle figure en bonne place sur la liste des européennes.

Fig. 4 Michèle Barzach à la une de Paris-Match, n¹982, 22 mai 1987.

\footnotetext{
33 TF1, 24 mars 1989, «Questions à domicile »; Le Figaro, 25 mars 1989, « Simone Veil pour une liste du centre $\gg$.

${ }^{34}$ Le Figaro, 24 avril 1989, « Simone Veil réussit son examen de passage ».
} 
Michèle Alliot-Marie (née en 1946) est elle secrétaire d’État chargée de l'Enseignement auprès du ministre de l'Éducation nationale et secrétaire nationale du RPR chargée des études et projets. Elle est à l'époque bien moins connue que Michèle Barzach.

Six listes obtiennent assez de suffrages pour avoir des députés à Strasbourg contre quatre lors des deux scrutins précédents ${ }^{35}$. Plus que la division de la droite sur deux listes, l'élément nouveau de cette élection est l'arrivée d'une importante délégation des Verts français au Parlement. Délégation comprenant près de la moitié de femmes. Comme le laissait prévoir la composition des listes, avec 18 élues, le scrutin européen de 1989 égale tout juste celui de 1979 pour le nombre de femmes parmi les députés européens français. Le RPR n’a que deux élues, comme en 1984.

Tableau 3 Les Françaises élues au Parlement européen en 1989 (par ordre de présentation sur les listes)

\begin{tabular}{|c|c|c|}
\hline Prénom Nom & $\begin{array}{c}\text { Date de } \\
\text { naissance }\end{array}$ & Antécédents européens \\
\hline \multicolumn{3}{|l|}{ Liste PS } \\
\hline Catherine Trautmann & 1951 & \\
\hline Nicole Péry & 1943 & $\begin{array}{l}\text { Députée européenne 1981-1984; élue en } \\
\text { 1984, première vice-présidente en } 1987\end{array}$ \\
\hline Martine Buron & 1944 & Députée européenne depuis 1988 \\
\hline Marie-Claude Vayssade & 1936 & $\begin{array}{c}\text { Elue en } 1979 \text { et 1984, députée européenne } \\
\text { 1979-1989, présidente de la commission } \\
\text { juridique } 1987-1989\end{array}$ \\
\hline Marie-Jo Denys & 1950 & \\
\hline Nora Zaïdi & 1965 & \\
\hline \multicolumn{3}{|l|}{ Liste des Verts } \\
\hline Solange Fernex & 1934 & \\
\hline Claire Schlecht-Joanny & 1951 & \\
\hline Marie-Christine Aulas & 1945 & \\
\hline Djida Tazdaït & 1957 & \\
\hline \multicolumn{3}{|l|}{ Liste PCF } \\
\hline Sylviane Einardi & 1948 & \\
\hline Sylvie Mayer (Le Roux) & 1947 & $\begin{array}{c}\text { Elue en 1979, députée européenne 1979-1984 } \\
\text { et depuis } 1986\end{array}$ \\
\hline Mireille Elmalan & 1949 & \\
\hline \multicolumn{3}{|l|}{ Liste du centre } \\
\hline Simone Veil & 1927 & $\begin{array}{l}\text { Elue en } 1979 \text { et } 1984 \text {, Présidente du } \\
\text { Parlement } 1979-1982 \text {, Présidente de la }\end{array}$ \\
\hline
\end{tabular}

\footnotetext{
${ }^{35}$ La liste d'Union UDF-RPR a obtenu 28,9\% des voix (26 élus) devant la liste socialiste (23,6 \%, 22 élus), la liste du Front National (11,7 \%, 10 élus) et la liste des Verts (10,6\%, 9 élus). La liste du centre conduite par Simone Veil arrive en cinquième position avec $8,4 \%$ des voix et 7 élus. Les communistes ont également 7 élus avec $7,71 \%$ des suffrages.
} 


\begin{tabular}{|c|c|c|}
\hline & & commission juridique 1982-1984 \\
\hline Nicole Fontaine & 1942 & Elue en 1984, députée européenne 1984-1989 \\
\hline Liste UDF-RPR & & \\
\hline Michèle Barzach (RPR) & $\mathbf{1 9 4 3}$ & \\
\hline Michèle Alliot-Marie (RPR) & $\mathbf{1 9 4 6}$ & \\
\hline Liste Front National & & \\
\hline Martine Lehideux & 1933 & Elue en 1984, députée européenne 1984-1989 \\
\hline
\end{tabular}

Michèle Alliot-Marie siège dans des commissions importantes : commission politique et commission des Affaires étrangères et de sécurité qui lui permettent d'acquérir une certaine compétence dans des domaines plutôt considérés comme masculins. Cela lui permet d'acquérir une expérience qu'elle met à profit en tant que secrétaire générale adjointe du RPR chargée des affaires étrangères (1990-1993). En même temps elle est députée de la $6^{\mathrm{e}}$ circonscription des Pyrénées-Atlantiques, ce qui ne facilite pas son engagement à Strasbourg. En mars 1993, elle démissionne de son mandat, venant d'être réélue députée et surtout nommée ministre de la Jeunesse et des Sports dans le gouvernement Balladur de cohabitation.

Pour Michèle Barzach, le mandat sera de courte durée. D'après Le Nouvel Observateur, dès l'élection, Jacques Chirac, président du RPR, téléphone à Michèle Barzach, et lui intime l'ordre de démissionner immédiatement afin qu'un de ses amis, moins bien placé sur la liste, puisse siéger à Strasbourg. Elle démissionne le 3 novembre 1989 et est remplacée par le suivant sur la liste, Aymeri de Montesquiou. Fezensac. Après son échec à entrer au conseil national du RPR, elle déclare : «je suis une femme qui dérange. Mais je crois surtout que je paie là mon combat pour l'union de l'opposition, pour cette force unique que réclament huit sur dix de nos électeurs ». Jacques Chirac, rappelle, quelque peu agacé : «Elle me doit sa carrière [...] J'en ai fait mon ministre de la Santé, mon adjoint à la mairie, et je l'ai imposée dans le quinzième arrondissement aux législatives et aux municipales ${ }^{36}$. » Elle dira de lui, citant Corneille : «il m'a fait trop bien pour que je puisse en dire du mal ; il m'a fait trop de mal pour que je puisse en dire du bien ». «Aucun affront, aucun rejet public ne m'est épargné, plus personne ne me salue, le chef a donné le signal, la meute peut se déchaîner ${ }^{37}$. »

\section{Conclusion}

Elues éphémères de la première législature en raison de l'application du «tourniquet »(1979-1984) ; femmes incarnant le gaullisme issu de la résistance ; femmes isolées car peu nombreuses et soucieuses de leur carrière politique en France, voici une courte

\footnotetext{
${ }^{36}$ Journal du Dimanche, 18 février 1990, Le Monde, 20 février 1990.

${ }^{37}$ Michèle Barzach, Vérités et tabous, Paris, Seuil, 1994.
} 
typologie des élues gaullistes au Parlement européen lors des trois premières élections européennes au suffrage universel. Pourtant le mode de scrutin avec listes nationales était propice à laisser, sinon encourager, les femmes accéder à une fonction politique moins courue par les hommes que les mandats nationaux. Mais les aléas de la vie politique et les échecs électoraux des gaullistes (1981 et 1988) ont fait du Parlement européen un sanctuaire où panser les plaies de la défaite et d'où pouvait partir une reconquête.

Hormis la personnalité exceptionnelle de Louise Weiss, qui siège à Strasbourg de 1979 à 1983, aucune autre élue portant l'étiquette « gaulliste » ne s'est imposée au Parlement européen pendant les trois premières législatures. Á la différence d'une Simone Veil, d'une Nicole Fontaine ou d'une Nicole Péry (PS). Sans doute leur destin politique européen individuel était-il forcément lié au positionnement du RPR sur les questions européennes.

Résumé en français :

En 1979, l'élection au suffrage universel du Parlement européen est une nouveauté qui ne plait guère aux gaullistes. Néanmoins, le RPR de Jacques Chirac doit mener campagne sans appétence particulière pour l'Europe. Figurant en cinquième position sur la liste gaulliste, Louise Weiss, «citoyenne de l'Europe », est comme une caution européenne au milieu de candidats qui entendent surtout défendre les intérêts de la France. Le tourniquet instauré par le RPR montre le peu d'importance qu'il donne à ce scrutin. Aux élections de 1984 et 1989, sur des listes au périmètre changeant, quelques femmes gaullistes sont élues mais force est de constater qu'elles ne jouent pas un grand rôle au sein de l'assemblée de Strasbourg.

Résumé en anglais :

The decision to elect representatives to the European Parliament through universal suffrage, starting in 1979, was a novelty that did not please the Gaullists in the least. Nevertheless, Jacques Chirac's RPR mounted a campaign despite its lack of real interest in the European election. Fifth on the Gaullist list, Louise Weiss, "citizen of Europe", ensured that the RPR could claim at least one genuine European advocate amidst candidates who intended to defend the interests of France above all. The turnstile of candidates established by the RPR showed what little importance it accorded to this vote. After managing to remain on the constantlychanging election lists of 1984 and 1989, some Gaullist women were elected but it was clear that they did not play a major role in the assembly in Strasbourg. 
Mots-clés : Louise Weiss ; femmes politiques ; gaullisme ; élections européennes ; Parlement européen

Keywords: Louise Weiss ; Women ; Gaullism ; European elections ; European Parliament

Yves Denéchère est professeur d'histoire contemporaine à l'Université d'Angers-UMR CERHIO. Un de ses axes de recherche porte sur l'engagement européen des femmes politiques françaises. Il a notamment publié : Ces Françaises qui ont fait l'Europe, Paris, Audibert, 2007. 